\title{
fondations spéciales et environnement
}

\author{
par \\ D. Gouvenot \\ SOLETANCHE Entreprise
}

\begin{abstract}
RESUME - Les techniques utilisées en matière de fondations spéciales (paroi moulée en béton armé, paroi d'étanchéité, injections, pieux forés et barrettes, etc...) ont reçu de très nombreuses applications lors des grands travaux de construction classique. Depuis quelques années, ces techniques participent à la protection de l'environnement, notamment par le fait qu'elles permettent la construction d'ouvrages enterrés dans des conditions géotechniques souvent difficiles. Grâce à ces procédés, on a pu concevoir et réaliser des stockages souterrains (hydrocarbures, gaz liquéfiés). Des écrans d'étanchéité ont pu être réalisés pour protéger les nappes phréatiques des eaux polluées ou des déchets industriels. Les injections de sol ont été utilisées à maintes reprises pour étancher et consolider les sols et permettre ainsi de protéger l'environnement des déchets et des effets des constructions humaines. Des progrès techniques certains ont été réalisés grâce à cette nouvelle orientation des fondations spéciales. De nouveaux matériaux ont dû être mis au point pour répondre à une protection de qualité de l'environnement.
\end{abstract}

\section{INTRODUCTION}

Utilisées traditionnellement pour résoudre les problèmes délicats de construction dans le sol, les techniques de fondations spéciales tendent de plus en plus à s'adapter à des applications spécifiques. Nous avons développé récemment $1^{\prime}$ application de ces techniques aux travaux maritimes (GOUVENOT, 1977).

Nous indiquerons, dans le présent article, les applications actuelles, résultant des efforts accomplis pour l'amélioration et la protection de l'environnement.

Les fondations spéciales qui comportent essentiellement des techniques de paroi moulée, de fondations forées, et d'injections, ont permis de résoudre des problèmes difficiles liés à la protection de l'environnement et ont apporté des solutions intéressantes aux problèmes suivants:

- Stockage souterrain des hydrocarbures : pétrole brut et gaz naturels liquéfiés.

- Protection des nappes souterraines en les isolant, à l'aide d'écrans étanches moulés dans le sol, de la pollution des déchets industriels ou ménagers.
- Fondation d'ouvrages, à l'aide des techniques de fondations forées, sur des zones de décharge, par utilisation de pieux injectés de haute capacité et de petit diamètre (pieux IM).

- Protection de l'environnement à l'aide des techniques de l'injection, par étanchement et consolidation des sols, pour éviter les mouvements de surface, au cours des travaux de creusement nécessité par l'exécution d'ouvrages souterrains.

Nous allons développer les deux premières applications en décrivant la technique utilisée, les matériaux mis au point pour développer ces techniques, des exemples de réalisation, et les problèmes de conception et d'exécution.

La troisième application fait l'objet de $l^{\prime}$ article $n^{\circ} 47$ du présent congrès.

La quatrième est citée pour mémoire.

LES STOCKAGES SOUTERRAINS A L'ABRI DE PAROIS MOULEES

Les parois moulées dans le sol constituent des enceintes en béton armé enterrées, à l'intérieur desquelles il est possible de 
réaliser des stockages très divers. Les avantages de ces stockages ont été déjà longuement développés et nous ne retiendrons que les plus marquants:

$1^{\circ}$ - Sécurité : les produits stockés sont en général dangereux (GNL par exemple), et les services de sécurité préfèrent souvent les voir stocker dans le sol. De plus, lorsque des réservoirs sont enterrés, la surface occupée au sol est considérablement réduite, car deux cuves souterraines peuvent être construites beaucoup plus rapprochées que des cuves aériennes.

$2^{\circ}$ - Esthétique : un stockage souterrain est en général peu visible ou invisible et réduit considérablement $1^{\prime}$ impact de ce genre de construction sur l'environnement.

$3^{\circ}$ - Coût : le coût de grands stockages souterrains est souvent plus intéressant que le stockage classique.

Nous allons décrire deux exemples de stockage: I'un se rapporte aux gaz naturels liquéfiés et a reçu de nombreuses applications, l'autre se rapporte au pétrole brut, et on prévoit, à l'heure actuelle, un essai semiindustriel, avant de passer à des réalisations en vraie grandeur.

Stockage souterrain de gaz naturels liquéfiés

Ce type de stockage se rapporte essentiellement au méthane liquide (GNL) pour lequel il existe déjà de nombreuses réalisations, notamment au Japon. Des études sont également en cours pour développer ce type de stockage dans les grands ports français pour du propane liquéfié (GPL).

La paroi moulée est utilisée pour permettre l'exécution de la fouille qui est en général cylindrique. Le diamètre et la profondeur de I'enceinte varient de 30 à $50 \mathrm{~m}$ environ (figure 1).

Après excavation à sec, la paroi est en général doublée par plusieurs enceintes (béton armé, isolant, acier, etc...) qui constituent la cuve du réservoir proprement dit.

Sur le plan géotechnique se pose le problème assez délicat du fonctionnement de la paroi cylindrique dans son rôle de soutènement provisoire puisque, dans cette phase, elle travaille simultanément en compression (dans un plan horizontal) du fait de l'effet voûte résultant de sa forme, et en flexion (dans un plan vertical) du fait de sa hauteur et de la diversité des sols qui peuvent être rencontrés. Des méthodes de dimensionnement ont été mises au point en tenant compte de la rigidité de l'anneau en béton que constitue la paroi d'une part, du comportement élastoplastique du sol, et des conditions d'appui particulières de la paroi, notamment en tête où elle est couronnée par une grosse cerce en béton, et dans le sol où elle trouve un appui par butée.

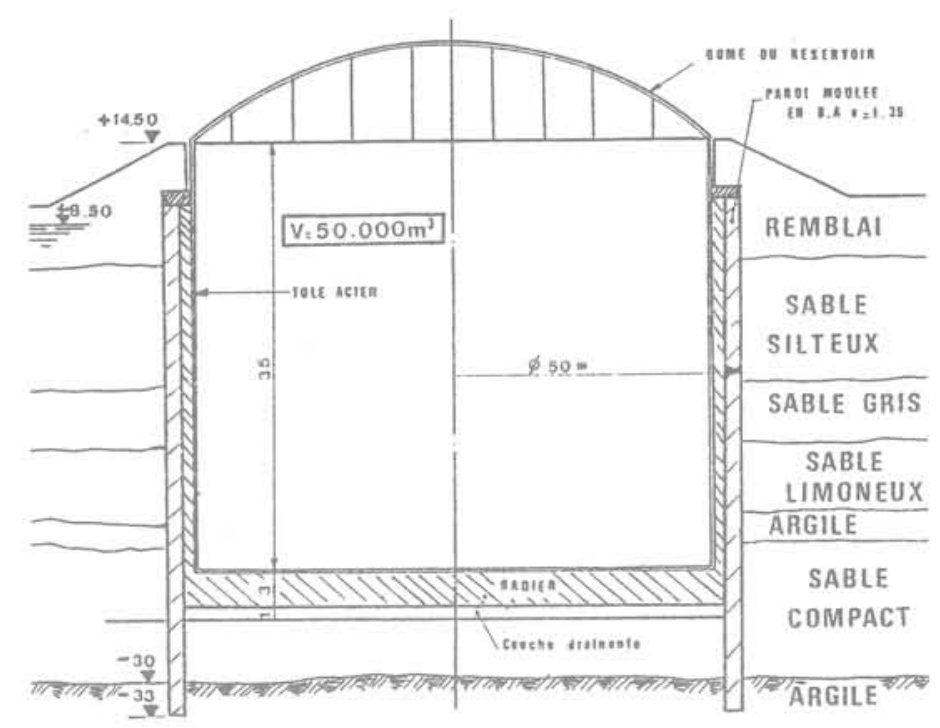

Figure 1: Réservoir de GNL en paroi moulée

Des problèmes très ardus sont également soulevés par la mise hors d'eau de la fouille, qui implique des études de stabilité d'ensemble vis-à-vis du glissement, et de stabilité du fond de fouille, aussi bien, en phase provisoire, qu'en phase d'exploitation du réservoir.

Selon les sols rencontrés, il y a trois types de réservoirs:

- Drainage temporaire du radier : ce type de disposition consiste à drainer le fond de fouille uniquement dans la phase de construction du réservoir. Elle est adoptée lorsque les sols sont suffisamment peu perméables pour qu'on puisse considérer qu'ils pourront geler en phase d'exploitation.

- Radier drainé : cette disposition vaut pour les sols plus perméables. La paroi allant s'ancrer dans un horizon étanche, l'eau provenant du sol est pompée sous le radier. De l'eau chaude est injectée sous le radier pour éviter le gel. Dans ces conditions, le radier peut être très léger et réduit à une dalle.

- Radier poids : cette technique est employée lorsqu'il n'y a pas d'horizon étanche. On peut réaliser un fond injecté pendant la phase de travaux et c'est un radier en béton très épais (de l'ordre de $5 \mathrm{~m}$ d'épaisseur) qui assure la stabilité du fond en phase d'exploitation.

On constate alors les exigences auxquelles doit satisfaire l'exécution de la paroi moulée :

$1^{\circ}$ - Respect de la forme géométrique circulaire pour limiter les contraintes dans le béton au taux du projet. Ceci implique des tolérances d'implantation et de verticalité 
difficiles, et qui sortent, en général, des spécifications usuelles.

$2^{\circ}$ - Qualité d'étanchéité de la paroi notamment en phase de travaux pour permettre la construction du réservoir à sec en assurant une qualité irréprochable des joints. L'utilisation d'appareils vérifiant la verticalité des panneaux en cours de perforation est souvent nécessaire pour s'assurer d'une bonne verticalité. Nous avons développé récemment un nouveau type de machines de perforation: l'hydrofraise, qui a été testée et est couramment utilisée au Japon, jusqu'd $100 \mathrm{~m}$ de profondeur, en respectant des spécifications de verticalité inférieures à ce qui est admis couramment pour des profondeurs nettement moins importantes.

L'influence du froid provoque un gel progressif du sol avec le temps qui peut durer plusi.eurs années. Ce gel provoque une bonne amélioration des caractéristiques du sol pour les sollicitations à court terme et notamment les sollicitations sismiques. Les contraintes induites par le gel et mesurées au Japon restent dans un domaine très acceptable (ISHIMASA, 1979).

Les mesures qui permettent de contrôler le bon fonctionnement de l'ouvrage en service consistent à suivre:

- La température du sol et de la paroi

(thermocouple).

- Les contraintes des aciers dans le béton (jauge de contrainte).

- La pression des terres (capteur de pression totale et de pression interstitielle).
- Les déplacements des terres résultant des gonflements dus au gel (clinomètre).

FUJITA (1979) donne les résultats de ce type de mesure pour un réservoir GNL de $45000 \mathrm{~m} 3$ construit au Japon.

L'intérêt des maitres d'oeuvre pour ce type de réservoir semble s'accroitre car il présente des avantages évidents vis-à-vis des problèmes de sécurité et d'environnement. Six de ces réservoirs sont déjà en service au Japon. Il s'en construit à l'heure actuelle de nombreux autres dans ce même pays. Plusieurs sont à l'étude en France et en Belgique.

Les problèmes d'exécution en ce qui concerne les fondations spéciales sont ardus, mais non insolubles, et nécessitent une instrumentation de surveillance qui est maintenant au point.

Stockage de pétrole brut dans une enceinte en paroi moulée (brevet PLASOL)

Pour résoudre les problèmes de stockage de pétrole brut posés par la mise en service des navires pétroliers de 200000 à 500000 tonnes, nous avons été amenés à étudier la réalisation de réservoirs semi enterrés de très grande capacité (de 200000 à 500000 m3 unitaires).

La technique utilisée pour construire la partie enterrée du réservoir est celle des parois moulées. La partie hors sol du réservoir et le toit flottant sont de construction classique en tôle d'acier. La partie hors sol est très réduite et rend ces réservoirs peu visibles.

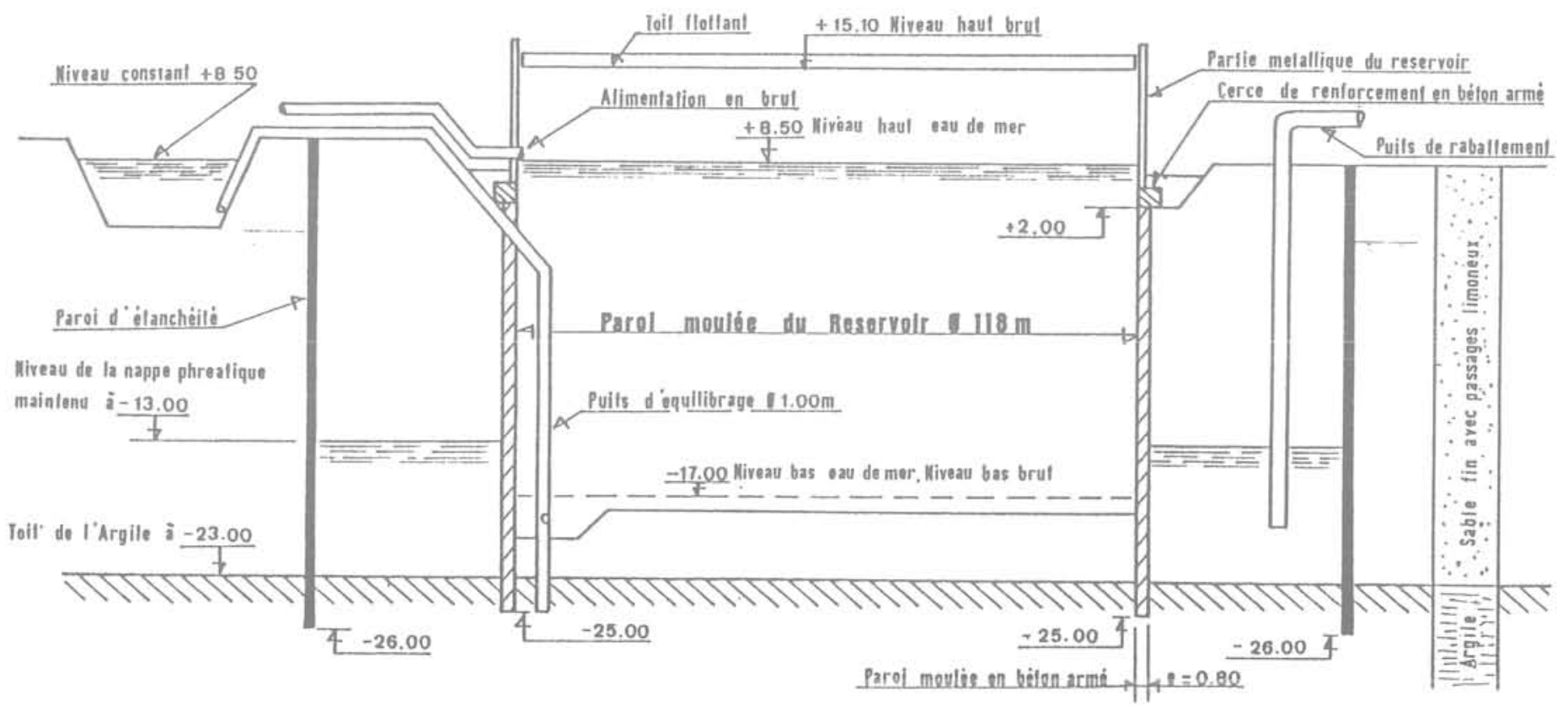

Figure 2 : Réservoir de type "PLASOL" pour hydrocarbures à base de paroi moulée 
Le principe de fonctionnement d'un tel réservoir consiste à équilibrer la pression du sol et de l'eau, extérieurs au réservoir, par la pression du fluide contenu. Ce fluide est tantôt le pétrole brut stocké, tantôt de l'eau mise en place dans le réservoir pour maintenir l'équilibre des pressions de part et d'autre de la jupe que constitue la paroi moulée.

Les phases de fonctionnement sont les suivantes:

$1^{\circ}$ - Réservoir plein d'eau : le réservoir est en communication avec un niveau d'eau constant par une tuyauterie. Ce niveau assure une pression constante sur le fond du réservoir dans toutes les phases d'exploitation. Le niveau constant est déterminé de telle façon que la paroi du réservoir enterré soit plaquée contre le sol afin d'assurer sa stabilité.

$2^{\circ}$ - Arrivée de pétrole brut : une conduite amène le pétrole au voisinage de la surface de l'eau.

$L^{\prime}$ interface eau-pétrole descend tandis que le niveau haut du pétrole monte dans le réservoir.

$3^{\circ}$ - Réservoir plein de pétrole : le procédé permet d'obtenir des réservoirs de très grande capacité avec bassin de rétention réduit puisque, seul, le volume d'hydrocarbures hors sol est à retenir, d'où, une faible occupation du sol. De plus, les pressions exercées sur la jupe du réservoir sont incomparablement plus faibles que pour un réservoir classique, d'où un gain sur le dimensionnement de la paroi du réservoir, et des possibilités de stockage de très grande dimension.

\section{Pringcipes_généraux de construction}

Contrairement au réservoir GNL décrit cidessus, le réservoir PLASOL ne peut jamais être vide, même en phase de travaux. La vidange provoquerait un déséquilibre de la paroi. On est donc conduit à excaver les terres situées à l'intérieur du réservoir, sous l'eau, ce qui nécessite d'utiliser des techniques de dragage. Le fond du réservoir ne peut donc être mis en place que sous l'eau. On utilise :

- Soit un fond naturel étanche (fond argileux par exemple).

- Soit un fond artificiel créé par injection ou par béton coulé sous l'eau.

Au niveau de la conception, on rencontre les mêmes problèmes que pour les réservoirs GNL, à savoir : étude d'une paroi circulaire sous des types de sollicitation complexes, stabilité du fond, stabilité d'ensemble aux grands glissements, etc...
Un écran étanche concentrique à la paroi moulée permet d'assurer la protection de la nappe phréatique naturelle. Nous reviendrons dans le paragraphe suivant sur cette technique. Cet écran étanche permet également d'abaisser en permanence le niveau d'eau à l'extérieur du réservoir à une cote telle que l'équilibre des pressions sur la jupe puisse être réalisé aisément. Le flambement de la jupe est à étudier avec soin, car l'on est souvent en présence d'une jupe en béton armé assez mince $(0,80 \mathrm{~m}$ à $1,20 \mathrm{~m})$, de très grand diamètre ( $100 \mathrm{~m}$ environ). On utilise les théries classiques de la résistance des matériaux. Comme, de plus, les pressions exercées sur la paroi sont, par hypothèse, assez faibles, il s'ensuit que l'on peut résoudre assez facilement la question du flambement en ajoutant une poutre de forte inertie à la tête de la paroi moulée.

Les problèmes d'exécution (implantation pour respecter la forme géométrique, verticalité, qualité des joints) sont aussi cruciaux que pour les réservoirs GNL.

Ce type de réservoir n'a pas été réalisé à ce jour. Nous préparons, pour l'instant, 10 réalisation d'un essai semi-industriel. Les études ont été menées en collaboration avec des sociétés pétrolières (brevet PLASOL) et ont montré qu'en dehors de ces avantages sur le plan sécurité, environnement, et facilité d'exploitation, ces techniques permettaient de réaliser des stockages de volume unitaire très important à un prix très inférieur et en utilisant des surfaces au sol beaucoup plus petites qu'arec un stockage classique. Les avantages pour l'environnement sont donc multiples.

LES ECRANS D'ETANCHEITE ET LA PROTECTION DES NAPPES PHREATIQUES

On a très souvent à protéger des nappes phréatiques des infiltrations d'eaux polluantes résultant soit de déchets industriels, soit de stockage d'eaux polluées. La technique des parois d'étanchéité, déjà connue pour ses applications en travaux hydrauliques (voile d'étanchéité dans les barrages, protection hydraulique des grandes fouilles excavées sous la nappe), répond très bien au problème de la protection des nappes phréatiques. Il est en effet possible de réaliser des "boites" étanches constituées par une paroi moulée périmétrale et un fond étanche naturel ou non (figure 3 ). 


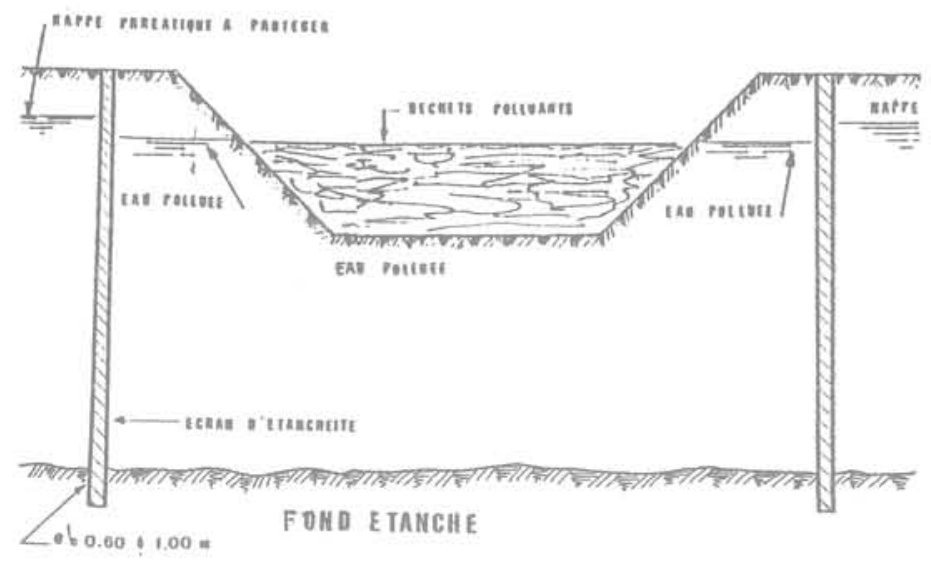

Figure 3 : Principe de stockage de déchets avec protection des nappes phréatiques

\section{Principe_de_la_protection}

On peut alors stocker des produits polluants ou des eaux polluées à l'intérieur de cette "boite". L'étanchéité de la paroi et du fond permet d'éviter les écoulements des fluides polluants et protège ainsi la nappe phréatique. Toutefois, l'étanchéité de ces "boîtes" n'étant jamais totale (nous reviendrons ultérieurement sur ce point), on peut prévoir des conditions d'utilisation qui protègent la nappe avec une sécurité absolve. Il suffit en effet d'assurer un niveau d'eau à l'intérieur de la "boite" inférieur au niveau de la nappe phréatique située d̀ l'extérieur. On élimine ainsi tout écoulement de l'intérieur vers l'extérieur, y compris à travers le fond. Les seuls écoulements possibles vont de l'extérieur vers l'intérieur. On élimine ainsi tout risque de pollution de la nappe.

Dans certaines conditions d'utilisation, on est amené à stocker les produits polluants à un niveau supérieur au niveau de la nappe. Il faut alors être capable d'estimer le débit de fuite pour pouvoir déterminer si le taux de produit polluant qui peut ainsi franchir les côtés de la "boite" ou son fond reste admissible pour la nappe phréatique.

\section{Iechnigues_utililisées}

La technique d'exécution de la paroi périmétrale peut être de deux types:

a) - Technique de la paroi moulée en béton armé, déjà citée pour les stockages souterrains.

b) - Technique de la paroi forée en continu sous un coulis auto durcissable.

Cette technique a été décrite par CARON (1973) et consiste à forer sous une bove bentonitique à laquelle on a ajouté du ci- ment. Le coulis reste fluide pendant la perforation puis durcit dans le sol et constitue ainsi l'écran étanche recherché. Cette technique est incomparablement plus économique que la technique de la paroi moulée en béton armé et permet une exécution en continu de la paroi. C'est la technique la mieux adaptée pour résoudre les problèmes d'étanchéité dans le sol sous gradient faible. L'écran étanche est donc un mélange de bentonite, de ciment, d'ajouts éventuels (filler par exemple) et d'adjuvants qui ont pour rôle, la plupart du temps, de régler le temps de prise du coulis. La perméabilité de tels écrans a été déterminée à partir des débits de percolation passant à travers des écrans de ce type et mesurés pour assurer un niveau constant à l'intérieur de la "botte". Les valeurs de perméabilité ainsi obtenues par application de la loi de DARCY conduisent en général à des valeurs de $10^{-7}$ $\mathrm{m} / \mathrm{s}$ (figure 4 ).

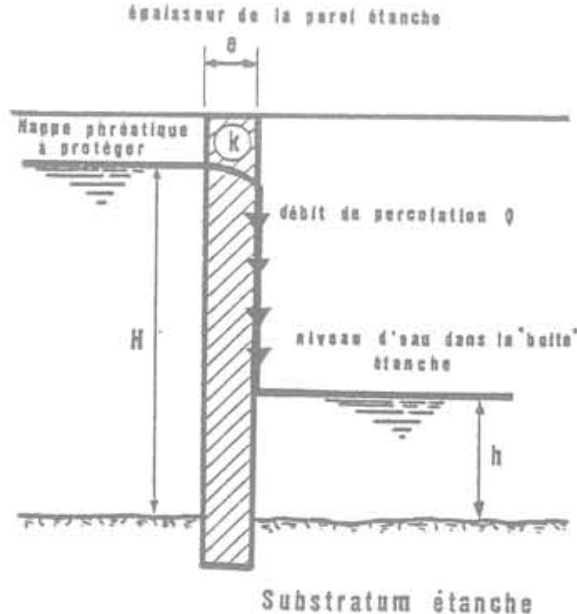

Q'enres Darey:

$$
\frac{x}{t}=\frac{20}{t} \frac{1}{x^{2}-x^{2}}
$$

Figure 4 : Détermination "en grand" de la perméabilité d'une enceinte étanche

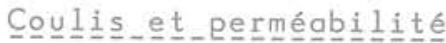

Cette valeur est obtenue pour les coulis usuels et a été testée sur des écrans de plusieurs hectares de surface développée. Cette perméabilité correspond à des débits de percolation de quelques $\mathrm{m} 3 / \mathrm{h}$ qui sont, la plupart du temps, acceptables sans gêner I'exploitation du stockage de déchets. Toutefois, dans le cas de déchets extrêmement polluants pour lesquels on n'admet que des débits de fuite très faibles sans pouvoir assurer un niveau intérieur suffisamment bas, on peut être conduit à rechercher des perméabilités plus petites. Notons que ceci ne présente d'intérêt que si la qualité du fond étanche est elle-même suffisante pour justi- 
fier la recherche d'une perméabilité réduite sur le voile périmétral. On peut être, alors, amené à augmenter les dosages usuels en ciment et en bentonite, ou même en filler, pour réduire la perméabilité, mais on aboutit assez rapidement à une impasse technique, car le coulis devient trop rigide et trop visqueux pour permettre une perforation de la tranchée dans des conditions acceptables. On utilise alors des dispersants qui améliorent la répartition de la matière sèche dans le volume du coulis et qui, en homogénéisant physiquement la structure du coulis, réduisent la perméabilité. On peut atteigdre ainsi des valeurs de perméabilité de 10 $\mathrm{m} / \mathrm{s}$ environ. Dans un cas de protection de nappes vis-à-vis de cations métalliques, il nous a été demandé des perméabilités encore plus petites. Ceci ne peut être réalisé économiquement et d'une manière techniquement satisfaisante qu'en travaillant sur la microstructure même du coulis. Il s'agit là d'une voie de très grand avenir qui n'a pas reçu encore d'application industrielle, mais qui permet d'espérer d'obtenir des coulis très étanches en améliorant la micro compacité du produit à $1^{\prime}$ échelle des cristaux de silicate de chaux qui se forment lors de l'hydratation et de la prise du coulis. Les photographies ci-dessous prises au microscope électronique à balayage montrent la différence de structure que l'on peut obtenir entre un coulis usuel et un coulis à microcompacité améliorée.

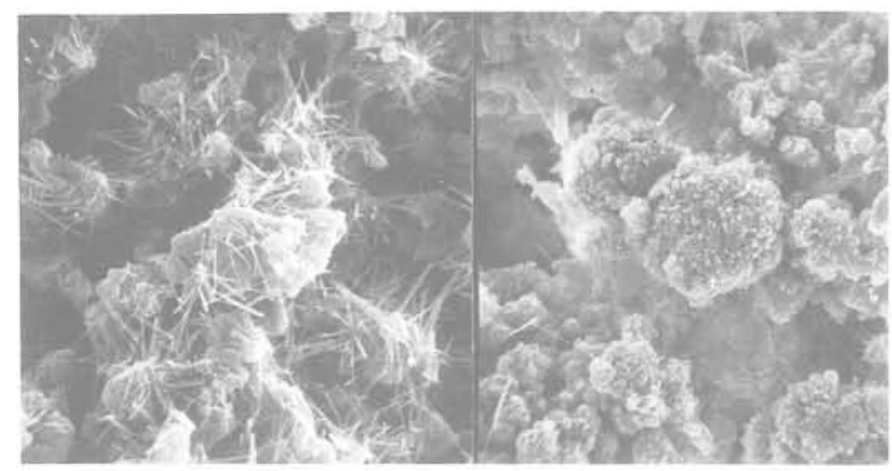

Figure 5

Coulis usuel bentonite-ciment
Coulis à microcompacité améliorée

N.B. : On remarque la différence de porosité entre les deux coulis qui sont pourtant à matière sèche égale (grossissement 2 500)

\section{Caractéristiques mécanigues des écrans}

Les caractéristiques du coulis d'étanchéité doivent être également examinées sur le plan de la résistance des matériaux et notamment sur la déformabilité de l'écran qui doit pouvoir subir les mouvements du sol lors de l'excavation de la fouille sans se fissurer. Il est courant de demander à ces coulis d'avoir un module d'Young voisin de celui du sol. En effet, ces écrans d'étanchéité peuvent être soumis à des déformations en tête de plusieurs centimètres. Il est incompatible d'exiger simultanément une perméabilité faible et un module d'Young bas. En effet, une perméabilité faible pour un coulis usuel ne peut s'obtenir qu'en augmentant les dosages de bentonite et de ciment. Ce qui se traduit par une résistance à la compression plus forte, et par un module d'Young plus fort. Les modules mesurés sur échantillon en compression simple sont de l'ordre de 1000 à 5000 bars. Ils sont donc en général beaucoup plus élevés que le module du sol en place. Pour traiter le problème correctement, il faut donc:

$1^{\circ}$ - Calculer la déformée du massif de sol situé en aval de l'écran d'étanchéité. A partir de cette déformée, il est possible de déterminer les contraintes réellement exercées dans l'écran. Ces contraintes résultent d'une part de la pesanteur et, d'autre part de la flexion imposée à l'écran par les déformations du sol. C'est la résultante de ces contraintes qui permet de fixer le module qu'il est raisonnable d'exiger du coulis, en écrivant la condition de traction nulle donc de non fissuration dans le coulis. Le module obtenu à partir de ces considérations peut être notablement plus élevé que celui du sol.

$2^{\circ}$ - On en déduit alors la résistance à la compression et la perméabilité du coulis.

Nous attirons l'attention sur le fait que ces paramètres ne sont pas indépendants. Si les valeurs trouvées ne sont pas compatibles avec les spécifications de l'utilisateur, il faut revenir à la condition d'utilisation avec niveau intérieur plus bas que le niveau extérieur. Les nouveaux coulis à microstructure améliorée ouvrent des horizons nouveaux car l'amélioration de la microstructure ne se fait pas par apport de matière sèche supplémentaire. Le choix du couple perméabilité-module sera plus large.

$3^{\circ}$ - Il convient de ne pas négliger l'étude des déformations localisées résultant de comportement différentiel, quand l'écran traverse un interface de deux couches de sol de rigidité différente (figure 6). 


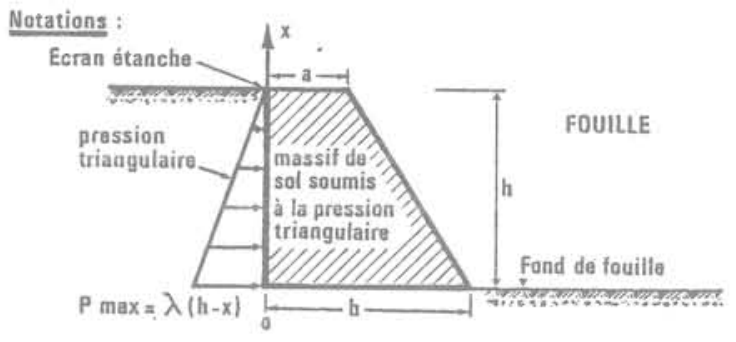

ba on pose : $c=\frac{b h}{b-a}$

Expression de la déformẻe : $y(x)$ :

$$
\begin{array}{r}
r(x)=\frac{2 \lambda}{E} \cdot\left(\frac{c}{b}\right)^{3} \times\left[\frac{(c-h)^{3}}{2 c}-\frac{(c-h)}{2 c^{2}}\left(11 c^{2}+4 c h-h^{2}\right)+\frac{x^{2}}{2}+\frac{(c-h)^{3}}{2(x-c)}\right. \\
\left.+3(c-h)(x-2 c+h) \cdot \log \frac{c-x}{c}\right] \\
-\frac{3 \lambda}{5 G} \cdot \frac{c}{b} \times\left[(c-2 h) \cdot x+\frac{x^{2}}{2}+(c-h)^{2} \cdot \log \frac{c-x}{c}\right]
\end{array}
$$

Figure 6a: Déformée d'ensemble d'un écran étanche

11 est important de vérifier la non fissuration, dans ces conditions, car il est courant de rencontrer des couches limoneuses ou vaseuses intercalées entre des couches sableuses. Lorsque le massif de sol se déforme, l'écran est soumis à des déformations différentielles qu'il ne peut absorber que si sa formulation en tient compte.

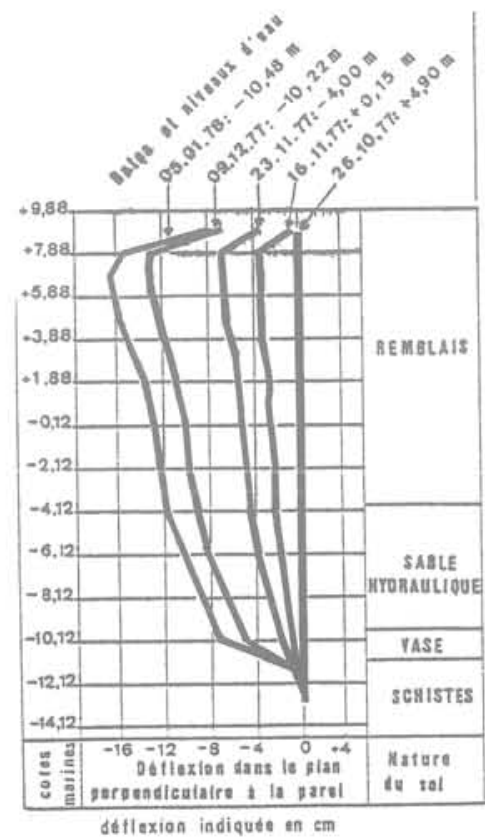

Figure 7 : Mesures clinométriques dans un écran d'étanchéité

On voit ainsi sur la figure 7 des mesures clinométriques réalisées, au sein d'un écran étanche, pendant les différentes phases

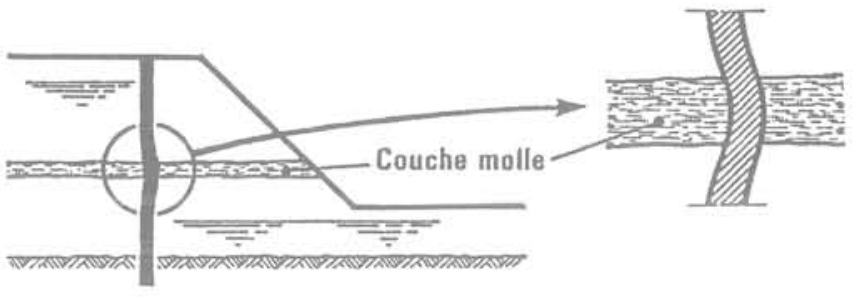

Couche molle intercalée

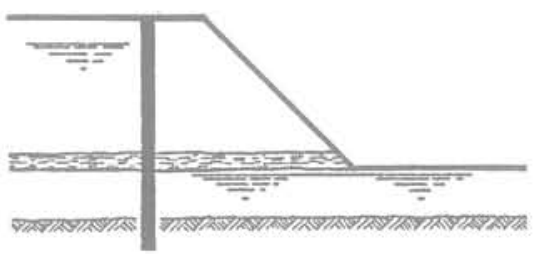

Couche molle à la base du "gabion"

Figure 6b: Déformée locale

de mise hors d'eau de la fouille. La déformée de l'écran est assez régulière, sauf au voisinage du fond de fouille, où elle a atteint plusieurs centimètres sur deux mètres de hauteur seulement, en raison de la présence d'une couche de vase à cet emplacement.

\section{Quglítéd_'exécutution}

Les problèmes d'exécution dépendent énormément de la nature du sous-sol. Il y a toutefois deux règles impérieuses à vérifier constamment :

$1^{\circ}$ - S'assurer que la plate-forme de travail est suffisamment haute par rapport aux différents niveaux piézométriques que $I^{\prime}$ on peut rencontrer dans le sous-sol ( 2 m environ), et il faut tenir compte de $l^{\prime}$ effet du marnage pour les travaux réalisés au voisinage d'un littoral.

$2^{\circ}$ - Vérifier en permanence la continuité des écrans pour assurer leur qualité d'étanchéité, car toute fenêtre peut être catastrophique, surtout dans un horizon perméable.

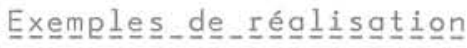

Les principales réalisations actuelles sont de deux types:

$1^{\circ}$ - Protection des nappes phréatiques autour des stockages d'hydrocarbures. 


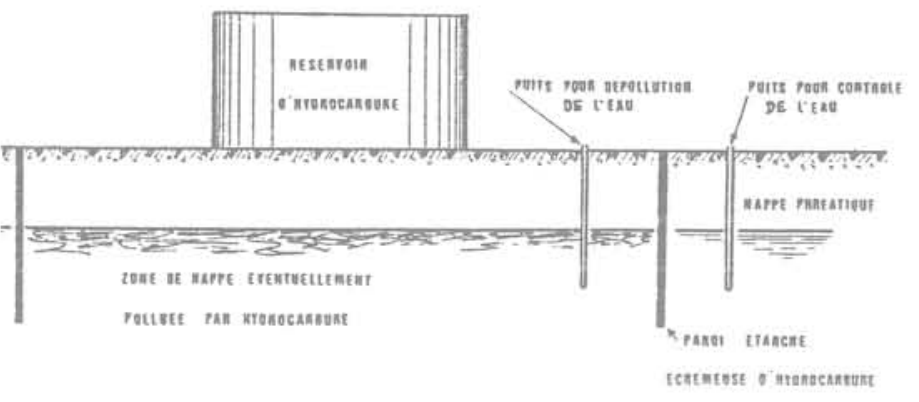

FOND ETANCHE

Figure 8 : Protection d'une nappe des hydrocarbures par un écran étanche

$2^{\circ}$ - Protection des nappes phréatiques autour des stockages de déchets industriels (voir figure 3 ).

Dans le cas de la protection vis-à-vis des hydrocarbures, une paroi "écrémeuse" suffit. En effet, les hydrocarbures ont une densité telle qu'ils surnagent et une paroi qui pénètre de deux mètres environ dans les nappes suffit à stopper leur progression. Il est possible de récupérer ces hydrocarbures par pompage dans la nappe polluée.

Nous avons réalisé ce type d'écran dans l'enceinte de très grandes raffineries.

Dans l'utilisation de protection totale de nappes par des déchets industriels, I'application a porté aussi bien sur des stériles miniers, que sur des matériaux de dragage fortement pollués par les rejets industriels.

Les mesures que l'on peut réaliser pour surveiller le fonctionnement de ces écrans consistent essentiellement à surveiller les différents niveaux d'eau à l'intérieur et à l'extérieur des "boîtes" étanches. On peut ainsi déceler à l'aide des piézomètres des anomalies dans l'écoulement des nappes.

D'autre part, les mesures de débit de percolation pendant la phase des travaux permettent d'avoir une très bonne idée de la perméabilité d'ensemble de la "boîte".

Enfin, il est recommandé de disposer de puits à l'extérieur de l'écran afin que des contrôles réguliers de l'eau puissent être réalisés à partir d'échantillons d'eau pompée à intervalles réguliers. En ce qui concerne la déformée des écrans, nous avons déjà cité l'utilisation de la clinométrie.

\section{CONCLUSIONS}

Les ouvrages enterrés destinés au stockage d'énergie (hydrocarbures) ou au stockage de déchets (stériles, déchets industriels ou ménagers) nécessitent une très grande qualité d'exécution, car tout défaut, notamment dans l'étanchéité conduit à des nuisances extrêmement graves sur l'environnement (pollution des nappes). Ceci explique le soin apporté dans l'amélioration des contrôles aussi bien en phase de travaux qu'en phase d'exploitation. L'ouvrage livré au stockage est ainsi vérifié et contrôlé avant d'être mis en service.

L'avantage apporté par les techniques de fondations spéciales concerne aussibien l'environnement de surface que l'environnement souterrain.

En surface :

- Sécurité améliorée par le stockage enterré ou semi enterré de produits dangereux.

- Nuisances réduites sur I'environnement par rapport aux stockages classiques en général peu esthétiques.

En profondeur :

- Protection des nappes phréatiques. A la paroi moulée, nous associons pour finir, les techniques d'injection utilisées depuis de nombreuses années pour prévenir les mouvements de surface lors du creusement de galeries, pour protéger les nappes, etc...

\section{REFERENCES}

Caron C., (1973). Un nouveau style de perforation; la bove autodurcissable. Annales de 1'ITBTP novembre 1973.

Fujita, (1979). Development and Operational Experiences of LNG in-ground storage tanks in Japan. Part. I I Osaka Gas Company 1979.

Gouvenot D., (1977). Les fondations spéciales en travaux maritimes.

Revue Travaux octobre 1977.

Ishimasa, (1979). Development and Operational Experiences of LNG in-ground storage tanks in Japan. Part. I Tokyo Gas Company 1979 . 